\title{
How to Derive Subdiffusion Equations?
}

\author{
T. KosztoŁOWICZ* \\ Institute of Physics, Jan Kochanowski University, Uniwersytecka 7, 25-406 Kielce, Poland \\ Doi: 10.12693/APhysPolA.139.314 *e-mail: tadeusz.kosztolowicz@ujk.edu.pl
}

\begin{abstract}
Processes of normal diffusion, subdiffusion, and slow subdiffusion (ultraslow diffusion) in a onedimensional homogeneous system are considered, all with or without absorption of diffusing particles. It is shown how to derive equations describing the processes. Subdiffusion equations contain the Caputo fractional time derivative while slow subdiffusion equations contain integral operators with kernels as slowly varying functions with respect to time. The Green functions for the equations are also presented.
\end{abstract}

topics: subdiffusion-reaction equations, fractional calculus

\section{Introduction}

Diffusion can be considered as a particle random walk in which particle jumps are described by the probability distributions of the jump length $\lambda(\rho)$ and the time the particle waits for the jump $\psi(\tau)[1-3]$. Usually, the kind of diffusion is defined by the relation $\left\langle(\Delta x)^{2}(t)\right\rangle \sim \xi(t)$. When $\xi(t)=t^{\alpha}$ we have superdiffusion for $\alpha>1$, normal diffusion for $\alpha=1$, and subdiffusion for $0<\alpha<1$. In turn, when $\xi$ is a slowly varying function, we have slow subdiffusion (ultraslow diffusion). A slowly varying function fulfills the condition $\xi(a u) / \xi(u) \rightarrow 1$ when $u \rightarrow \infty$ for any positive $a$. A slowly varying functions are combinations of logarithmic functions or functions having finite limit at infinity. In the case of superdiffusion, a particle can perform anomalously long jumps, for example in a medium in which turbulence occurs. The variation of such jump length is infinity whereas $\langle\tau\rangle$ is finite. For normal diffusion, both variables $\rho$ and $\tau$ have finite moments. In the case of subdiffusion, the first moment of the waiting time for a jump is infinite whereas the moments of length of a jump are finite. When a diffusing particle can be absorbed or undergo irreversible chemical reactions, the probability of the particle disappearing depends on the type of diffusion.

In the following, we consider the normal diffusion, subdiffusion, and slow subdiffusion processes, all with and without absorption. Subdiffusion occurs in media in which diffusion of particles is very hindered due to a complex structure of the medium. For example, subdiffusion can occur when sugars are transported in a gel [4]. Subdiffusion with absorption has been observed, e.g., when antibiotics diffuse in a bacterial biofilm $[5,6]$. The bacterial defence mechanisms can either permanently retain the antibiotic molecules in the biofilm or cause the molecules to break down.
In this paper, it is shown how to derive the equations describing the mentioned diffusion processes, using a particle random walk model in a onedimensional homogeneous discrete system. The type of diffusion is related to the appropriate choice of the function $\psi$. The Green functions for the derived equations are also shown. The Green function $P(x, t)$ is a probability density of finding a particle at $x$ at time $t$. It is the solution of a diffusion equation with the initial condition $P(x, 0)=\delta\left(x-x_{0}\right)$, where $\delta$ is the delta-Dirac function, $x_{0}$ is the initial location of the particle.

\section{Model}

We use a simple model of a particle random walk in a discrete system. Let us consider the difference equation describing a particle random walk in the system

$$
P_{n+1}(x)=\frac{1}{2} P_{n}(x-\Delta x)+\frac{1}{2} P_{n}(x+\Delta x),
$$

where $P_{n}(x)$ is the probability that the particle is in the position $x$ performing $n$ steps and $\Delta x$ is the length of a particle jump. In order to move from discrete to continuous time, we use the relation $P(x, t)=\sum_{n=0}^{\infty} P_{n}(x) \Phi_{n}(t)$. Here, $P_{n}(x)$ is the probability that a particle, starting from $x_{0}$, reaches site $x$ in $n$ steps, and $\Phi_{n}(t)$ is the probability that the particle performs $n$ steps in the time interval $(0, t)[7]$. The latter probability can be found using

$$
\begin{aligned}
& \Phi_{n}(t)=\left(U * Q_{n}\right)(t), \quad Q_{i}(t)=\left(Q_{i-1} * \psi\right)(t), \\
& Q_{0}(t)=\delta(t),
\end{aligned}
$$

$i \geq 1$, where $U(t)$ is the probability that the particle has not performed any step over a time interval $(0, t), Q_{i}(t)$ is the probability density that the $i$-th particle jump has been made at time $t$. The symbol $*$ denotes the convolution of functions, i.e., $(f * g)(t)=\int_{0}^{t} \mathrm{~d} t^{\prime} f\left(t-t^{\prime}\right) g\left(t^{\prime}\right)$. 
It is convenient to use the Laplace transform $\mathcal{L}[f(t)]=\int_{0}^{\infty} \mathrm{d} t \mathrm{e}^{-s t} f(t)=\hat{f}(s)$ in further considerations. As $\mathcal{L}[(f * g)(t)]=\hat{f}(s) \hat{g}(s)$, we can write that $\hat{\Phi}_{n}(s)=\hat{U}(s) \hat{Q}_{n}(s)=\hat{U}(s) \hat{\psi}^{n}(s)$. The difference equations generating function is $S(x, z)=$ $\sum_{n=0}^{\infty} z^{n} P_{n}(x)$. Combining it with the above equations leads to $\hat{P}(x, s)=\hat{U}(s) S(x, \hat{\psi}(s))$. In the limit of small $\Delta$, one can use the approximation $(\hat{P}(x+\Delta x, s)+\hat{P}(x-\Delta x, s)-2 P(x, s)) /(\Delta x)^{2} \approx$ $\frac{\partial^{2} \hat{P}(x, s)}{\partial x^{2}}$. Now, from the definition of the generating function $S(x, z)$, when changing $z \rightarrow \hat{\psi}(s)$, and from (1), we derive

$$
\hat{\psi}(s)\left(S(x, \hat{\psi}(s))-P_{0}(x)\right)=\frac{\partial^{2} S(x, \hat{\psi}(s))}{\partial x^{2}} .
$$

Since $S(x, \hat{\psi}(s))=\frac{\hat{P}(x, s)}{\hat{U}(s)}$, we get

$$
\frac{1-\hat{\psi}(s)}{\hat{U}(s)} \hat{P}(x, s)-P(x, 0)=\frac{(\Delta x)^{2} \hat{\psi}(s)}{2 \hat{U}(s)} \frac{\partial^{2} \hat{P}(x, s)}{\partial x^{2}} \text {. }
$$

For a given $\hat{\psi}(s)$ and then computing $\hat{U}(s)$, we get the diffusion equation given in terms of the Laplace transform. The absorption or decay process of a diffusing particle can be included in the function $\hat{\psi}(s)[8]$.

\section{Diffusion without absorption}

If there is no absorption of diffusing particles, the normalization condition $\int_{0}^{\infty} \mathrm{d} t \psi(t)=1$ is satisfied leading to the condition $\hat{\psi}(0)=1$. In this case, one has $\hat{\psi}(s)=\left(1+\frac{v(s)(\Delta x)^{2}}{2 D}\right)^{-1}[9,10]$, where the function $v(s)$ defines a kind of diffusion, $v(s) \rightarrow 0$ when $s \rightarrow 0$, and $D$ is the generalized diffusion coefficient. We have $U(t)=1-\int_{0}^{t} \mathrm{~d} t^{\prime} \psi\left(t^{\prime}\right)$. Using the formulae $\mathcal{L}[1]=\frac{1}{s}$ and $\mathcal{L}\left[\int_{0}^{t} \mathrm{~d} t^{\prime} f\left(t^{\prime}\right)\right]=\frac{\hat{f}(s)}{s}$, we obtain $\hat{U}(s)=\frac{1-\hat{\psi}(s)}{s}$. In the limit of small $\Delta x$, we have $\hat{\psi}(s) \approx 1-\frac{v(s)(\Delta x)^{2}}{2 D}$ and $\hat{U}(s) \approx \frac{v(s)(\Delta x)^{2}}{2 s D}$. Combining (4) with the above equations, we get

$$
\frac{v(s)}{s}(s \hat{P}(x, s)-P(x, 0))=D \frac{\partial^{2} \hat{P}(x, s)}{\partial x^{2}} .
$$

The solution of (5) for the initial condition $P(x, 0)=\delta\left(x-x_{0}\right)$ and boundary conditions $\hat{P}( \pm \infty, s)=0$ (these boundary conditions are assumed for all cases considered in this paper) is

$$
\hat{P}(x, s)=\frac{1}{2 s} \sqrt{\frac{v(s)}{D}} \exp \left(-\sqrt{\frac{v(s)}{D}}\left|x-x_{0}\right|\right) .
$$

The time evolution of the mean square displacement reads

$$
\begin{aligned}
& \left\langle(\Delta x)^{2}(t)\right\rangle= \\
& \int_{-\infty}^{\infty} \mathrm{d} x\left(x-x_{0}\right)^{2} P(x, t)=\mathcal{L}^{-1}\left[\frac{2 D}{s v(s)}\right]
\end{aligned}
$$

As mentioned above, the type of diffusion is determined by the function $v(s)$. For its classification, we use moments of the fractional order of the distribution $\psi$, defined as $\left\langle\tau^{\rho}\right\rangle=\int_{0}^{\infty} \mathrm{d} \tau \tau^{\rho} \psi(\tau)$, $\rho>0$ [10]. These fractional moment can be calculated in terms of the Laplace transform,

$$
\mathcal{L}\left[\left\langle\tau^{\rho}(t)\right\rangle\right]=\frac{(-1)^{k}}{\Gamma^{k}(k-\rho)^{k}} \int_{0}^{\infty} \mathrm{d} s s^{k-\rho-1} \frac{\mathrm{d}^{k} \hat{\psi}(s)}{\mathrm{d} s^{k}},
$$

where $k$ is the smallest natural number such that $k>\rho$. For the moment of natural order $\rho=k$, (8) takes the following form $\mathcal{L}\left[\left\langle\tau^{k}\right\rangle\right]=$ $(-1)^{k} \mathrm{~d}^{k} \hat{\psi}(s) /\left.\mathrm{d} s^{k}\right|_{s=0}$.

\subsection{Normal diffusion}

Normal diffusion can be defined as a particle random walk process in which the mean value of the waiting time for a particle jump is finite. In this case, one assumes $v(s)=s$, which provides to the relation $\left\langle(\Delta x)^{2}(t)\right\rangle=2 D t$. Knowing that $\mathcal{L}^{-1}[s \hat{P}(x, s)-P(x, 0)]=\partial P(x, t) / \partial t$, the normal diffusion equation is

$$
\frac{\partial P(x, t)}{\partial t}=D \frac{\partial^{2} P(x, t)}{\partial x^{2}} .
$$

Next, using $\mathcal{L}^{-1}\left[\frac{1}{\sqrt{s}} \mathrm{e}^{-\sqrt{s}|x| / \sqrt{D}}\right]=\frac{1}{\sqrt{\pi t}} \mathrm{e}^{-x^{2} / 4 D t}$, from (6) we get

$$
P(x, t)=\frac{1}{2 \sqrt{\pi D t}} \exp \left(-\frac{\left(x-x_{0}\right)^{2}}{4 D t}\right) .
$$

\subsection{Subdiffusion}

In the subdiffusive particle random walk, there is $\langle\tau\rangle=\infty$. However, there exists a positive constant $\alpha<1$ such that fractional moments fulfill the conditions $\left\langle\tau^{\rho}\right\rangle<\infty$ for $0<\rho \leq \alpha$ and $\left\langle\tau^{\rho}\right\rangle=\infty$ for $\rho>\alpha$. These relations are met when $v(s)=s^{\alpha}$, $0<\alpha<1$. Now, applying the following relation

$$
\mathcal{L}^{-1}\left[\frac{1}{s^{\nu+1}}\right]=\frac{t^{\nu}}{\Gamma(1+\nu)}
$$

into (7), where $\nu>-1$ and $\Gamma(1+\nu)$ denotes the gamma function, we obtain $\left\langle(\Delta x)^{2}(t)\right\rangle=\frac{2 D t^{\alpha}}{\Gamma(1+\alpha)}$. Using $\mathcal{L}^{-1}\left[s^{\alpha} \hat{g}(s)-s^{\alpha-1} g(0)\right]=\partial_{C}^{\alpha} g(t) / \partial t^{\alpha}$ in (5), we obtain

$$
\frac{\partial_{C}^{\alpha} P(x, t)}{\partial t^{\alpha}}=D \frac{\partial^{2} P(x, t)}{\partial x^{2}} .
$$

Here, the Caputo fractional derivative is defined for $0<\alpha<1$ as

$$
\begin{aligned}
& \frac{\partial_{C}^{\alpha} g(t)}{\partial t^{\alpha}}=\frac{1}{\Gamma(1-\alpha)} \int_{0}^{t} \mathrm{~d} t^{\prime}\left(t-t^{\prime}\right)^{-\alpha} \frac{\mathrm{d} f\left(t^{\prime}\right)}{\mathrm{d} t^{\prime}} . \\
& \text { Using } \mathcal{L}^{-1}\left[s^{\nu} \mathrm{e}^{-a s^{\beta}}\right]=f_{\nu, \beta}(t ; a)[11] \text { with } \\
& f_{\nu, \beta}(t ; a)=t^{-1-\nu} \sum_{k=0}^{\infty} \frac{1}{k ! \Gamma(-\nu-\beta k)}\left(-\frac{a}{t^{\beta}}\right)^{k},
\end{aligned}
$$


where $a, \beta>0$ (the function $f_{\nu, \beta}$ is a particular case of the Fox H-function and the Wright function), from (6) we get

$$
P(x, t)=\frac{1}{2 \sqrt{D}} f_{-1+\alpha / 2, \alpha / 2}\left(t ; \frac{\left|x-x_{0}\right|}{D}\right) .
$$

\subsection{Slow subdiffusion}

Slow subdiffusion (ultraslow diffusion) can be defined as a particle random walk process in which all positive fractional moments are infinite, $\left\langle\tau^{\rho}\right\rangle=\infty$, $\rho>0$. If $v(s)$ is a slowly varying function, $\psi(s)$ generates the above relation [12]. The diffusion equation and the Green function depend on the specific form of $v(s)$. To calculate the inverse Laplace transform of the Green function, we use the strong Tauberian theorem [13].

This Tauberian theorem states: if $g(t) \geq 0$, $g(t)$ is ultimately monotonic as $t \rightarrow \infty, R$ is a slowly varying function at infinity and $0<\eta<\infty$, then each of the relations (i) $\hat{g}(s) \approx R(1 / s) / s^{\eta}$ as $s \rightarrow 0$ and $\left(\right.$ ii) $g(t) \approx R(t) / \Gamma(\eta) t^{1-\eta}$ as $t \rightarrow \infty$, implies the other. Due to this theorem, we get in the long time limit

$$
P(x, t)=\frac{1}{2} \sqrt{\frac{v(1 / t)}{D}} \exp \left(-\sqrt{\frac{v(1 / t)}{D}}\left|x-x_{0}\right|\right) .
$$

For example, assuming $v(s)=\left(\log ^{2 r}(1 / s)\right)^{-1}$, $r>0$, and further using $\mathcal{L}^{-1}\left[(1 / s) \log ^{2 r}(1 / s)\right]=$ $\mu(t, 2 r-1) / \Gamma(2 r)[14]$, we obtain [15]

$$
\frac{1}{\Gamma(r)} \int_{0}^{t} \mathrm{~d} t^{\prime} \mu(t, r) \frac{\partial P\left(x, t^{\prime}\right)}{\partial t^{\prime}}=D \frac{\partial^{2} P(x, t)}{\partial x^{2}},
$$

where $\mu(t, b)=\int_{0}^{\infty} \mathrm{d} z t^{z} z^{b} / \Gamma(1+z)$ is the Volterratype function [16]. In the long time limit, we get

$$
\begin{aligned}
& P(x, t)=\frac{1}{2 \sqrt{D} \log ^{r}(t)} \\
& \quad \times \exp \left(-\frac{1}{\log ^{r}(t)} \sqrt{\frac{\left|x-x_{0}\right|}{D}}\right) .
\end{aligned}
$$

Sample plots of the Green functions for normal diffusion (10), subdiffusion (15), and slow subdiffusion (18) are shown in Fig. 1.

\section{Diffusion with absorption}

We can distinguish two processes that lead to the disappearance of a diffusing particle with a probability that does not change with time. The first is the decay of the diffusing particle $A$, which corresponds to the reaction $A \rightarrow B$. In this case, the probability of the reaction does not depend on the position of the particle. In the second process, the particle has to meet the "absorption center" $B$, after which a reaction can occur with a certain probability. Absorption can be treated here as the reaction $A+B \rightarrow B$. Let $p$ be the probability that

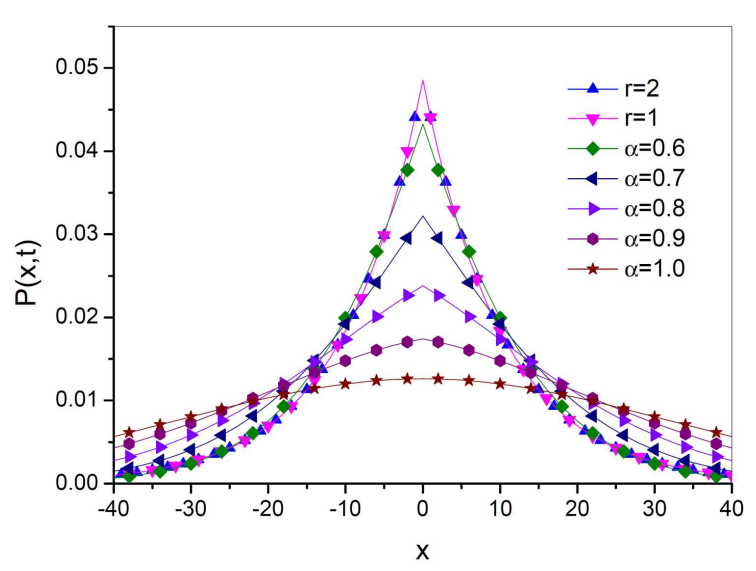

Fig. 1. The plots of Green's function for slow subdiffusion are marked by $r$ in the legend, for subdiffusion by $\alpha, \alpha=1.0$ corresponds to normal diffusion, the other parameters are $D=5, x_{0}=0$, and $t=100$; all parameters are given in arbitrarily chosen units.

the particle will meet the absorption center after the jump. Assuming that $B$ are homogeneously distributed in the system, $p$ does not depend on the spatial variable. For reaction $A \rightarrow B$, there is $p=1$. Let the decay probability density of the molecule that met $B$ be $\phi(t)=\gamma \mathrm{e}^{-\gamma t}, \gamma$ is a coefficient controlling a decay process. The probability that the molecule will not decay in the time interval $(0, t)$ is $1-\int_{0}^{t} \mathrm{~d} t^{\prime} \phi\left(t^{\prime}\right)=\mathrm{e}^{-\gamma t}$. The probability density $\psi_{R}(t)$ of the particle to jump to a new position after time $t$ depends on the probability that the particle has not decayed by that time.

A similar remark concerns the probability $U_{R}(t)$ that a particle will not change its position up to time $t$. We have

$$
\psi_{R}(t)=(1-p) \psi(t)+p \mathrm{e}^{-\gamma t} \psi(t)
$$

and

$$
U_{R}(t)=\left(1-\left(1-\mathrm{e}^{-\gamma t}\right) p\right)\left(1-\int_{0}^{t} \mathrm{~d} t^{\prime} \psi\left(t^{\prime}\right)\right) .
$$

Due to the formula $\mathcal{L}\left[\mathrm{e}^{-\gamma t} f(t)\right]=\hat{f}(s+\gamma)$, we get

$$
\begin{aligned}
& \hat{\psi}_{R}(s)=(1-p) \hat{\psi}(s)+p \hat{\psi}(s+\gamma) \\
& \hat{U}_{R}(s)=(1-p) \frac{1-\hat{\psi}(s)}{s}+p \frac{1-\hat{\psi}(s+\gamma)}{s+\gamma} .
\end{aligned}
$$

Change of $\hat{\psi} \rightarrow \hat{\psi}_{R}$ and $\hat{U} \rightarrow \hat{U}_{R}$ in (4) and use of an approximation $\hat{\psi}_{R}(s) \approx 1-$ $\frac{(\Delta x)^{2}}{2 D}(v(s)+v(s+\gamma))$ provide to

$$
\begin{aligned}
& D \frac{\partial^{2} \hat{P}(x, s)}{\partial x^{2}}=(1-p) \frac{v(s)}{s}(s \hat{P}(x, s)-P(x, 0)) \\
& \quad+p \frac{v(s+\gamma)}{s+\gamma}((s+\gamma) \hat{P}(x, s)-P(x, 0)) . \quad(23)
\end{aligned}
$$


The solution of $(23)$ is

$$
\begin{aligned}
& \hat{P}(x, s)=\frac{1}{2 \sqrt{D}} \frac{(1-p) \frac{v(s)}{s}+p \frac{v(s+\gamma)}{s+\gamma}}{\sqrt{(1-p) v(s)+p v(s+\gamma)}} \\
& \times \exp \left(-\frac{\sqrt{(1-p) v(s)+p v(s+\gamma)}}{\sqrt{D}}\left|x-x_{0}\right|\right) .
\end{aligned}
$$

and can be obtained by means of the Fourier transform method. In the time domain, one deals with

$$
\begin{aligned}
& D \frac{\partial^{2} P(x, t)}{\partial x^{2}}=(1-p) \int_{0}^{t} \mathrm{~d} t^{\prime} F\left(t-t^{\prime}\right) \frac{\partial P\left(x, t^{\prime}\right)}{\partial t^{\prime}} \\
& +p \mathrm{e}^{-\gamma t} \int_{0}^{t} \mathrm{~d} t^{\prime} F\left(t-t^{\prime}\right) \frac{\partial\left(\mathrm{e}^{\gamma t^{\prime}} P\left(x, t^{\prime}\right)\right)}{\partial t^{\prime}},
\end{aligned}
$$

where $F(t)=\mathcal{L}^{-1}[v(s) / s][8]$.

$$
\text { 4.1. The case of } p=1
$$

For $p=1$, the probability that a particle is at point $x$ at time $t$ can be obtained using the Green function for the system without absorption multiplied by the probability of absorption over the time interval $(0, t)$. In this case, the solution reads [17]:

$$
P(x, t)=\mathrm{e}^{-\gamma t} P_{\gamma=0}(x, t),
$$

where $P_{\gamma=0}$ is the solution in the system without particle decay, which is given by the inverse Laplace transform of (6).

\subsection{The case of $p<1$}

\subsubsection{Normal diffusion}

For $v(s)=s,(21)-(24)$ provide

$$
\begin{aligned}
& s \hat{P}(x, s)-P(x, 0)=D \frac{\partial^{2} \hat{P}(x, s)}{\partial x^{2}}-p \gamma \hat{P}(x, s) \\
& \hat{P}(x, s)=\frac{1}{2 \sqrt{D(s+p \gamma)}} \\
& \quad \times \exp \left(-\sqrt{\frac{s+p \gamma}{D}}\left|x-x_{0}\right|\right)
\end{aligned}
$$

With the use of (9) and (10) (see Sect. 3.1), we can write that in the time domain one has

$$
\begin{aligned}
& \frac{\partial P(x, t)}{\partial t}=D \frac{\partial^{2} P(x, t)}{\partial x^{2}}-p \gamma P(x, t), \\
& P(x, t)=\frac{\mathrm{e}^{-p \gamma t}}{2 \sqrt{\pi D t}} \exp \left(-\frac{\left(x-x_{0}\right)^{2}}{4 D t}\right) .
\end{aligned}
$$

\subsubsection{Subdiffusion}

When $v(s)=s^{\alpha}, 0<\alpha<1,(23)$ are not really appropriate to practical use. For this reason, we consider (23) in the limit of small $s$, which corresponds to the long time limit in the time domain.
Assuming $s \ll \gamma$, which corresponds to $t \gg 1 / \gamma$, we get the following approximations of (23) and (24), respectively:

$$
\begin{aligned}
& (1-p)(s \hat{P}(x, s)-P(x, 0))= \\
& s^{1-\alpha}\left(D \frac{\partial^{2} \hat{P}(x, s)}{\partial x^{2}}-p \gamma^{\alpha} \hat{P}(x, s)\right), \\
& \hat{P}(x, s)=\frac{s^{\alpha-1}}{2 \kappa \sqrt{\widetilde{D}}} \exp \left(-\frac{\kappa}{\sqrt{\widetilde{D}}}\left|x-x_{0}\right|\right) \\
& \quad \times \exp \left(-\frac{(1-p) s^{\alpha}}{2 \kappa \sqrt{\widetilde{D}}}\left|x-x_{0}\right|\right),
\end{aligned}
$$

where $\widetilde{D}=D /(1-p)$ and $\kappa=\sqrt{p \gamma^{\alpha} /(1-p)}$. In the time domain, we get

$$
\begin{aligned}
& \frac{\partial_{C}^{\alpha} P(x, t)}{\partial t^{\alpha}}=D \frac{\partial^{2} P(x, t)}{\partial x^{2}}-\kappa^{2} P(x, t) . \\
& P(x, t)=\frac{\mathrm{e}^{-\left|x-x_{0}\right| \kappa / \sqrt{\widetilde{D}}}}{2 \kappa \sqrt{\widetilde{D}}} f_{-1+\alpha, \alpha}\left(t ; \frac{\left|x-x_{0}\right|}{2 \kappa \widetilde{D}}\right) .
\end{aligned}
$$

\subsubsection{Slow subdiffusion}

For $v(s)=\ln ^{-2 r}\left(\frac{1}{s}\right)$, when $r>0$, we obtain (25) with $F(t)=\mu(t, 2 r-1) / \Gamma(2 r)$. However, strong Tauberian theorem cannot be applied to (24) unless we properly approximate (23). Such considerations are rather complicated and will be presented elsewhere.

\section{Final remarks and conclusions}

The methods of deriving the normal diffusion, subdiffusion, and slow subdiffusion equations describing processes without absorption and with absorption of diffusing particles have been shown. To derive the equations, we use the model of a particle random walk in a discrete system. This model is particularly useful for deriving the Green function and membrane boundary conditions for normal diffusion, subdiffusion, and slow subdiffusion in a system with a thin membrane [18-20]. The subdiffusion equations considered in this paper contain the fractional Caputo derivative of the order $\alpha$ with respect to time. The more commonly used form is the fractional Riemann-Liouville derivative which for $0<\beta<1$ is defined as

$$
\frac{\mathrm{d}_{R L}^{\beta} g(t)}{\mathrm{d} t^{\beta}}=\frac{1}{\Gamma(1-\beta)} \frac{\mathrm{d}}{\mathrm{d} t} \int_{0}^{t} \mathrm{~d} t^{\prime}\left(t-t^{\prime}\right)^{-\beta} g\left(t^{\prime}\right) .
$$

The subdiffusion equation reads

$$
\frac{\partial P(x, t)}{\partial t}=D \frac{\partial_{R L}^{1-\alpha}}{\partial t^{1-\alpha}} \frac{\partial^{2} P(x, t)}{\partial x^{2}} .
$$

Both forms of the subdiffusion equation with different fractional time derivatives are equivalent to each other. However, the Caputo derivative has some 
properties "closer" to the "ordinary" derivative. For example, the Caputo derivative of a constant function is zero whereas the Riemann-Liouville derivative does not have this property.

In general, there are many definitions of fractional derivatives not equivalent to each other [21]. We can call the fractional derivative the operator dependent on the continuous parameter $\beta$, which for $\beta=1,2, \ldots$ takes the form of an "ordinary" derivative of the natural order, but for $\beta \notin N$ these operators may not be equivalent to each other. The Grünwald-Letnikov fractional derivative, which can be treated as a generalization of the limit of the differential quotient, is equivalent to the Riemann-Liouville derivative. The GrünwaldLetnikov derivative is most often used in the numerical solving of subdiffusion equations, see for example [22]. We mention that superdiffusion can be described by the equation with the fractional Riesz derivative with respect to a spatial variable.

The subdiffusion equations presented in this paper are fractional parabolic equations containing at most a first-order time derivative. However, one can derive hyperbolic subdiffusion equations containing time derivatives of the order $\beta \in(1,2]$. Such an equation can be derived using a persistent random walk of a particle model, for which the direction of the previous particle jump is preferred in its next jump due to the particle inertia. For example, for normal diffusion we can assume that the flux is lagged with respect to the gradient of $P(x, t)$, thus $J(x, t+\tau)=-D \partial P(x, t) / \partial x$, where $\tau$ is a small parameter given in a time unit. Approximating the flux as $J(x, t+\tau) \approx J(x, t)+\tau \partial J(x, t) / \partial t$ and combining the above equations with the continuity equation $\partial P / \partial t=-\partial J / \partial x$, we get the hyperbolic diffusion equation $\tau \partial^{2} P / \partial t^{2}+\partial P / \partial t=$ $D \partial^{2} P / \partial x^{2}$ [23]. The hyperbolic subdiffusion equation is not chosen unambiguously [24].

In practice, irrespective of the form of the hyperbolic subdiffusion equation, solutions to the parabolic and hyperbolic equations in a homogeneous system or in a membrane system usually differ very little, such that the easier to solve parabolic one is chosen to be used. However, there are subdiffusion and normal diffusion processes for which the use of these equations gives qualitatively different results, even for a small parameter $\tau$. Examples of this are: subdiffusive impedance [25] and subdiffusion-reaction process in which the terms of the reaction depend on the parameter $\tau$ [26].

\section{References}

[1] R. Metzler, J. Klafter, Phys. Rep. 339, 1 (2000).

[2] R. Metzler, J. Klafter, J. Phys. A 37, R161 (2004).
[3] J. Klafter, I.M. Sokolov, First Step in Random Walks. From Tools to Applications, Oxford UP, New York 2011.

[4] T. Kosztołowicz, K. Dworecki, S. Mrówczyński, Phys. Rev. Lett. 94, 170602 (2005).

[5] T. Kosztołowicz, R. Metzler, Phys. Rev. E 102, 032408 (2020).

[6] T. Kosztołowicz, R. Metzler, S. Wąsik, M. Arabski, PLoS One 15, e0243003 (2020).

[7] E.W. Montroll, G.H. Weiss, J. Math. Phys. 6, 167 (1965).

[8] T. Kosztołowicz, K.D. Lewandowska, Phys. Rev. E 90, 032136 (2014).

[9] T. Kosztołowicz, Phys. Rev. E 91, 022102 (2015).

[10] T. Kosztołowicz, Phys. Rev. E 99, 022127 (2019).

[11] T. Kosztołowicz, J. Phys. A 37, 10779 (2004).

[12] S.I. Denisov, H. Kantz, Phys. Rev. E 83, 041132 (2011).

[13] B.D. Hughes, Random Walk and Random Environments. Vol. I, Random Walks, Clarendon, Oxford 1995.

[14] F. Oberhettinger, F. Badii, Tables of Laplace Transforms, Springer, Berlin 1973.

[15] T. Kosztołowicz, J. Stat. Mech. P10021, 1 (2015).

[16] R. Garrappa, F. Mainardi, Analysis 36, 89 (2015).

[17] I.M. Sokolov, M.G.W. Schmidt, F. Sagués, Phys. Rev. E 73, 031102 (2006).

[18] T. Kosztołowicz, Int. J. Heat Mass Transfer 111, 1322 (2017).

[19] T. Kosztołowicz, Physica A 298, 285 (2001).

[20] T. Kosztołowicz, A. Dutkiewicz, Math. Meth. Appl. Sci. 43, 10500 (2020).

[21] I. Podlubny, Fractional Differential Equations, Academic, San Diego 1999.

[22] T. Kosztołowicz, K.D. Lewandowska, Phys. Rev. E 78, 066103 (2008).

[23] G. Cattaneo, Atti Semin. Mat. Fis. Univ. Modena 3, 83 (1948).

[24] A. Compte, R. Metzler, J. Phys. A 30, 7277 (1997).

[25] T. Kosztołowicz, K.D. Lewandowska, J. Phys. A 42, 055004 (2009).

[26] T. Kosztołowicz, Phys. Rev. E 90, 042151 (2014). 